\title{
Nonexplosion and Pathwise Uniqueness of Stochastic Differential Equation Driven by Continuous Semimartingale with Non-Lipschitz Coefficients
}

\author{
Jinxia Wang \\ College of Science, Xian University of Technology, Xian, Shanxi 710048, China \\ Correspondence should be addressed to Jinxia Wang; wangjx@xaut.edu.cn
}

Received 7 October 2014; Revised 22 March 2015; Accepted 9 April 2015

Academic Editor: Niansheng Tang

Copyright (C) 2015 Jinxia Wang. This is an open access article distributed under the Creative Commons Attribution License, which permits unrestricted use, distribution, and reproduction in any medium, provided the original work is properly cited.

We study a class of stochastic differential equations driven by semimartingale with non-Lipschitz coefficients. New sufficient conditions on the strong uniqueness and the nonexplosion are derived for $d$-dimensional stochastic differential equations on $\mathbb{R}^{d}(d>2)$ with non-Lipschitz coefficients, which extend and improve Fei's results.

\section{Introduction}

Consider the following stochastic differential equations driven by a nonlinear integrator (or semimartingale) of the form

$$
\begin{aligned}
d X_{t} & =x_{0}+\int_{0}^{t} F\left(X_{s}, d s\right) \\
& =x_{0}+\int_{0}^{t} b\left(X_{s}, s\right) d s+\int_{0}^{t} M\left(X_{s}, d s\right),
\end{aligned}
$$

equivalently

$$
d X_{t}=b\left(X_{t}, t\right) d t+M\left(X_{t}, d t\right), \quad X_{0}=x_{0},
$$

where $(\Omega, \mathscr{F}, P)$ is a probability space, $\left(\mathscr{F}_{t}\right)_{t \geqslant 0}$ is $\sigma$ fields, $b(t, \cdot, \cdot)$ is a $d$-dimensional process of finite variation and $\mathscr{F} \times$ $\mathscr{B}\left(W^{d}\right) / \mathscr{B}\left(\mathbb{R}^{d}\right)$ adapted function, $M(x, t)$ is a $d$-dimensional continuous local martingale, and the pair $(a(x, y, t), b(x, t))$ is the local characteristic of $F(x, t)$ (for details, see Mao [1]).

Recently, many studies have focused on the strong uniqueness and the nonexplosion of stochastic differential equations with the coefficients satisfying the local Lipchitz condition (see Stroock and Varadhan [2] and Krylov [3]). However, the results on the strong uniqueness of stochastic differential equations are still very few when the coefficients satisfy non-Lipschitz condition, except the one-dimensional case (for details, see Ikeda and Watanabe [4] and Revuz and Yor [5]). Let $X_{t}\left(x_{0}, \omega\right)$ be the solution of the stochastic differential equations (1). If the driving process $M(x, t)$ is only the Brownian motion and local characteristic is locally Lipschitzian, Protter proved that the solution admitted a continuous version $\widetilde{X}_{t}\left(x_{0}, \omega\right)$ (see [6]). When the drift term is independent of $t$, satisfying local $L_{q}-L_{p}$ integrated condition, and the diffusion term is identity matrix, Krylov and Röckner proved the existence and path uniqueness for stochastic differential equations on a given area (see [7]). From the viewpoint of application, to what extent the condition on coefficients should be weakened is an important problem. Fang and Zhang discussed the pathwise uniqueness and the nonexplosion for a class of stochastic differential equations driven by Brownian motion with non-Lipschitz coefficients (see [8]). Using Zvonkin's transformation, Zhang studied the homeomorphic property of solutions of multidimensional stochastic differential equations with non-Lipschitz coefficients (see [9]). When the diffusion coefficient is uniformly nondegenerate and non-Lipschitz and drift coefficient is locally integrable, Zhang also proved the existence of a unique strong solution up to the explosion time for a stochastic differential equation. Moreover, two nonexplosion conditions are given (see [10]). Davie proved the uniqueness of solutions of stochastic differential equations when the drift term is bounded Borel function and the diffusion term is identity 
matrix (see [11]). Using Gronwall lemma, Fei studied the existence and uniqueness of solutions for fuzzy random differential equations with non-Lipschitz coefficients and discussed the dependence of fuzzy random differential equations on initial values (see [12]). Luo studied the behaviors of small subsets under the flows generated by the ordinary and stochastic differential equations whose coefficients satisfy certain non-Lipschitz conditions (see [13]). Further, for the stochastic differential equations with non-Lipschitz coefficients driven by Brown motion, Lan proved the pathwise uniqueness and nonexplosion, deriving the new sufficient condition (see [14]). Note that there are very few results about the case of semimartingale. Recently, Fei proved that the solution of the stochastic differential equations (1) was not exploding, when the local characterized $a$ and $b$ satisfy the following conditions:

$$
\begin{gathered}
a(x, x, t) \leqslant C\left(|x|^{2} \rho\left(|x|^{2}\right)+1\right), \\
|b(x, t)| \leqslant C\left(|x| \rho\left(|x|^{2}\right)+1\right),
\end{gathered}
$$

where $C>0$ is a constant and $\rho \in C^{1}([1,+\infty])$ is a strictly positive function which satisfies the following conditions:

(i) $\lim _{s \rightarrow+\infty} \rho(s)=+\infty$.

(ii) $\int_{1}^{\infty}(1 /(s \rho(s)+1)) d s=+\infty$.

(iii) $\lim _{s \rightarrow+\infty}\left(s \rho^{\prime}(s) / \rho(s)\right)=0$.

And the pathwise uniqueness of the stochastic differential equations (1) is also proved, under the following conditions:

$$
\begin{aligned}
& a(x, x, t)-2 a(x, y, t)+a(y, y, t) \\
& \quad \leqslant C|x-y|^{2} \lambda\left(|x-y|^{2}\right), \\
& |b(x, t)-b(y, t)| \leqslant C|x-y| \lambda\left(|x-y|^{2}\right),
\end{aligned}
$$

where $\lambda:\left[0, \delta_{0}\right] \rightarrow \mathbb{R}^{+}$is a strictly positive $C^{1}$ function satisfying the conditions

(i) $\lim _{s \rightarrow 0} \lambda(s)=+\infty$;

(ii) $\int_{0}^{\delta_{0}}(1 / s \lambda(s)) d s=+\infty$;

(iii) $\lim _{s \rightarrow 0}\left(s \lambda^{\prime}(s) / \lambda(s)\right)=0$.

For details, see [15]. Ulteriorly, Cao proved the existence and pathwise uniqueness of solutions of SDE driven by class of special semimartingale when the coefficient of martingale term is non-Lipschitz and satisfies Taniguchi's condition (see [16]) and Yang established the pathwise uniqueness of SPDE with non-Lipschitz coefficients (see [17]).

In this paper, we derive the new sufficient conditions on the strong uniqueness and the nonexplosion for $d$ dimensional stochastic differential equations (1) driven by semimartingale with non-Lipschitz coefficients on $\mathbb{R}^{d}(d>$ 2 ), and the new conditions are sharp in a sense. The theory of related martingale would be found in $[4,5,18]$.

This paper is organized as follows. In Section 2, we derive the new sufficient conditions and prove the nonexplosion for stochastic differential equations (1) on $\mathbb{R}^{d}(d>2)$. In Section 3, we prove the pathwise uniqueness under some new sufficient conditions and stochastic continuity.

\section{No Explosion of Solutions}

In this section, we prove the following nonexplosion result of stochastic differential equations (1). Let $X_{t}(\omega)$ be the solution of the stochastic differential equations (1) and

$$
\begin{gathered}
a(x, y, t) \in C\left(\mathbb{R}^{d} \times \mathbb{R}^{d} \times \mathbb{R} ; \mathbb{R}^{+}\right), \\
b(x, t) \in C\left(\mathbb{R}^{d} \times \mathbb{R} ; \mathbb{R}\right) .
\end{gathered}
$$

$\zeta$ is the lifetime of solution process $X_{t}$.

Theorem 1. Let $\gamma:[0, \infty) \rightarrow \mathbb{R}^{+}$be a $C^{1}$ function which satisfies the following conditions:

(i) $\lim _{s \rightarrow \infty} \gamma(s)=\infty$.

(ii) $\int_{0}^{\infty}(1 /(\gamma(s)+1)) d s=\infty$.

(iii) for any $s \in[M, \infty)$, there exists $M>1$ such that $\gamma^{\prime}(s) \geqslant 0,2 \gamma(s) / s-\gamma^{\prime}(s) \geqslant 0$.

$\Lambda: \Omega \times \mathbb{R} \rightarrow \mathbb{R}$ is a positive and progressive measurable function which satisfies

$$
\mathbb{E} \int_{0}^{t} \Lambda(s, \omega) d s<\infty, \quad t \geqslant 0 .
$$

If the condition

$$
a(x, x, t)+2\langle x, b(t, x)\rangle \leqslant \Lambda(t, \omega)\left(\gamma\left(|x|^{2}\right)+1\right)
$$

holds, then the stochastic differential equations (1) have no explosion $P(\zeta=+\infty)=1$.

Proof. Define

$$
\tilde{\gamma}(x)= \begin{cases}x^{2} \gamma\left(\frac{1}{x}\right), & 0<x<1, \\ \gamma(x), & x \geqslant 1 .\end{cases}
$$

According to the continuity of $a$ and $b$, there exists $\Lambda \geqslant 0$ such that condition (7) holds with respect to $\tilde{\gamma}(x)$ for any $x$. Let $\Gamma(x)=\int_{0}^{x}(1 /(\widetilde{\gamma}(s)+1)) d s ;$ then $\Gamma^{\prime}(x)=1 /(\widetilde{\gamma}(x)+1)$,

$$
\Gamma^{\prime \prime}(x)= \begin{cases}-\frac{\left(2 x \gamma(1 / x)-\gamma^{\prime}(1 / x)\right)}{\left(x^{2} \gamma(1 / x)+1\right)^{2}}, & 0<x<1, \\ \frac{-\gamma^{\prime}(x)}{(\gamma(x)+1)^{2}}, & x \geqslant 1 .\end{cases}
$$

Due to (iii) of condition (7), we know that there exists $M>1$ such that $\Gamma^{\prime \prime}(x) \leqslant 0$ for $x<0$ or $x>M$. It is obvious that the function $\Gamma^{\prime \prime}(x)$ is not continuous at the point $x=1$; hence the Itô formula is not applied for $\Gamma$. Now take $\widetilde{\Gamma} \in C^{2}(R)$ such that $\widetilde{\Gamma}(x) \geqslant \Gamma(x)$, and

$$
\widetilde{\Gamma}(x)=\Gamma(x), \quad \text { for } x<\frac{1}{M} \text { or } x>M .
$$


In fact, $\Gamma^{\prime \prime}(x)$ exists for any $x \neq 1$; we can take

$$
\begin{aligned}
& \widetilde{\Gamma}(x) \\
& = \begin{cases}\in C^{2}\left(\left[\frac{1}{M}, M\right]\right), \widetilde{\Gamma} \geqslant \Gamma, & x \in\left[\frac{1}{M}, M\right], \\
\Gamma(x), & x<\frac{1}{M} \text { or } x>M\end{cases}
\end{aligned}
$$

and $\widetilde{\Gamma}^{\prime \prime}(1 / M)=\Gamma^{\prime \prime}(1 / M), \widetilde{\Gamma}^{\prime \prime}(M)=\Gamma^{\prime \prime}(M)$.

Let $\xi_{t}(\omega)=\left|X_{t}(\omega)\right|^{2}$; define

$$
\begin{gathered}
K=\sup _{x \in[1 / M, M]}\left(\left|\Gamma^{\prime}(x)\right|+\left|\Gamma^{\prime \prime}(x)\right|\right), \\
\tau_{R}=\inf \left\{t>0, \xi_{t} \geqslant R\right\}, \quad R>0 ;
\end{gathered}
$$

then $\tau_{R} \uparrow \zeta$ as $R \uparrow \infty$. Let

$$
I_{\omega}=\left\{t>0, \xi_{t}(\omega) \in\left[\frac{1}{M}, M\right]\right\} .
$$

If $s \in I_{\omega}$, then we have

$$
a\left(X_{s}, X_{s}, s\right)+2\left\langle X_{s}, b\left(s, X_{s}\right)\right\rangle \leqslant C_{M} \Lambda(s, \omega),
$$

where $C_{M}=\sup _{x \in[1 / M, M]}(\gamma(x)+1)$. Note that

$$
\begin{aligned}
d \xi_{t}= & 2 X_{t} M\left(X_{t}, d t\right)+2 X_{t} b\left(X_{t}, t\right) d t \\
& +a\left(X_{t}, X_{t}, t\right) d t
\end{aligned}
$$

and the stochastic covariation $d \xi_{t} \cdot d \xi_{t}$ is given by

$$
d \xi_{t} \cdot d \xi_{t}=4 a\left(X_{t}, X_{t}, t\right) X_{t}^{2} d t .
$$

By the Itô formula and (15) and (16), we have

$$
\begin{aligned}
\widetilde{\Gamma}\left(\xi_{t \wedge \tau_{R}}\right)= & \widetilde{\Gamma}\left(\xi_{0}\right)+2 \int_{0}^{t \wedge \tau_{R}} \widetilde{\Gamma}^{\prime}\left(\xi_{s}\right)\left\langle X_{s}, M\left(X_{s}, d s\right)\right\rangle \\
& +2 \int_{0}^{t \wedge \tau_{R}} \widetilde{\Gamma}^{\prime}\left(\xi_{s}\right)\left\langle X_{s}, b\left(X_{s}, s\right)\right\rangle d s \\
& +\int_{0}^{t \wedge \tau_{R}} \widetilde{\Gamma}^{\prime}\left(\xi_{s}\right) a\left(X_{s}, X_{s}, s\right) d s \\
& +2 \int_{0}^{t \wedge \tau_{R}} \widetilde{\Gamma}^{\prime \prime}\left(\xi_{s}\right) a\left(X_{s}, X_{s}, s\right) X_{s}^{2} d s .
\end{aligned}
$$

Taking expectation for (17) and according to (7) and (14), we have

$$
\begin{aligned}
& \mathbb{E} \widetilde{\Gamma}\left(\xi_{t \wedge \tau_{R}}\right)=\widetilde{\Gamma}\left(\xi_{0}\right)+\mathbb{E}\left(\int_{0}^{t \wedge \tau_{R}} \widetilde{\Gamma}^{\prime}\left(\xi_{s}\right)\right. \\
& \left.\quad \cdot\left(2\left\langle X_{s}, b\left(X_{s}, s\right)\right\rangle+a\left(X_{s}, X_{s}, s\right)\right) d s\right) \\
& \quad+2 \mathbb{E}\left(\int_{0}^{t \wedge \tau_{R}} \widetilde{\Gamma}^{\prime \prime}\left(\xi_{s}\right) a\left(X_{s}, X_{s}, s\right) X_{s}^{2} d s\right):=\widetilde{\Gamma}\left(\xi_{0}\right) \\
& +J_{1}+J_{2} .
\end{aligned}
$$

For $J_{1}$, due to (14), we have

$$
\begin{aligned}
J_{1} & =\mathbb{E}\left(\int_{0}^{t \wedge \tau_{R}} \mathbb{q}_{I_{\omega}}(s) \widetilde{\Gamma}^{\prime}\left(\xi_{s}\right)\right. \\
& \left.\cdot\left(2\left\langle X_{s}, b\left(X_{s}, s\right)\right\rangle+a\left(X_{s}, X_{s}, s\right)\right) d s\right) \\
& +\mathbb{E}\left(\int_{0}^{t \wedge \tau_{R}} \mathbb{\square}_{I_{\omega}^{c}}(s) \widetilde{\Gamma}^{\prime}\left(\xi_{s}\right)\right. \\
& \left.\cdot\left(2\left\langle X_{s}, b\left(X_{s}, s\right)\right\rangle+a\left(X_{s}, X_{s}, s\right)\right) d s\right) \\
& \leqslant K C_{M} \mathbb{E}\left(\int_{0}^{t \wedge \tau_{R}} \Lambda(s, \omega) d s\right) \\
& +\mathbb{E}\left(\int_{0}^{t \wedge \tau_{R}} \frac{1}{\tilde{\gamma}\left(\xi_{s}\right)+1} \Lambda(s, \omega) \widetilde{\gamma}\left(\xi_{s}\right) d s\right) .
\end{aligned}
$$

For $J_{2}$, noting that $\Gamma^{\prime \prime}(x) \leqslant 0$ for $x<0$ or $x>M$, we have

$$
\begin{aligned}
J_{2}= & 2 \mathbb{E}\left(\int_{0}^{t \wedge \tau_{R}} \mathbb{}_{I_{\omega}}(s) \widetilde{\Gamma}^{\prime \prime}\left(\xi_{s}\right) a\left(X_{s}, X_{s}, s\right) X_{s}^{2} d s\right) \\
& +2 \mathbb{E}\left(\int_{0}^{t \wedge \tau_{R}} \mathbb{\square}_{I_{\omega}^{c}}(s) \widetilde{\Gamma}^{\prime \prime}\left(\xi_{s}\right) a\left(X_{s}, X_{s}, s\right) X_{s}^{2} d s\right) \\
\leqslant & 2 \mathbb{E}\left(\int_{0}^{t \wedge \tau_{R}} \mathbb{\square}_{I_{\omega}}(s) \widetilde{\Gamma}^{\prime \prime}\left(\xi_{s}\right) a\left(X_{s}, X_{s}, s\right) X_{s}^{2} d s\right) \\
\leqslant & 2 K M C_{M} \mathbb{E}\left(\int_{0}^{t \wedge \tau_{R}} \Lambda(s, \omega) d s\right) .
\end{aligned}
$$

Combining (19) and (20), it holds that

$$
\begin{aligned}
\mathbb{E} \widetilde{\Gamma}\left(\xi_{t \wedge \tau_{R}}\right) & \\
\leqslant & \widetilde{\Gamma}\left(\xi_{0}\right) \\
& +\left(1+K C_{M}+2 K M C_{M}\right) \mathbb{E}\left(\int_{0}^{t \wedge \tau_{R}} \Lambda(s, \omega) d s\right) \\
& :=C_{t}<\infty .
\end{aligned}
$$

Hence,

$$
\mathbb{E} \Gamma\left(\xi_{t \wedge \tau_{R}}\right) \leqslant \mathbb{E} \widetilde{\Gamma}\left(\xi_{t \wedge \tau_{R}}\right) \leqslant C_{t}<\infty .
$$

Letting $R \uparrow \infty$, by the Fatou lemma, we have

$$
\mathbb{E} \int_{0}^{\xi_{t \wedge \zeta}} \frac{1}{\tilde{\gamma}(x)+1} d s \leqslant C_{t}<\infty .
$$

If $P(\zeta<\infty)>0$, then there exists $T>0$ such that $P(\zeta<$ $T)>0$; take $t=T$ in (23); we have $\mathbb{E}\left(\mathbb{\square}_{\zeta \leqslant T} \Gamma\left(\xi_{\zeta}\right)\right) \leqslant C_{t}$, which is impossible, because of $\Gamma\left(\xi_{\zeta}\right)=\infty$.

\section{Pathwise Uniqueness of Solutions}

In this section, we will prove the following pathwise uniqueness result; when the driving process is Brownian motion, such kind of properties was studied for non-Lipschitzian coefficients in [8]. 
Theorem 2. Let $\eta:[0,1) \rightarrow \mathbb{R}^{+}, \eta(0)=0$, be a positive and continuous function on some intersection $[0, \epsilon)(\epsilon>0)$, and $\int_{0}^{+\infty}(1 / \eta(s)) d s=\infty$ and $\Theta: \Omega \times \mathbb{R} \rightarrow \mathbb{R}$ is a positive and progressive measurable function satisfying

$$
\mathbb{E} \int_{0}^{t} \Theta(s, \omega) d s<\infty, \quad t>0 .
$$

If $a$ and $b$ satisfy the condition

$$
\begin{aligned}
a(x, x, t)-2 a(x, y, t)+a(y, y, t) \\
+2\langle x-y, b(t, x)-b(t, y)\rangle \\
\leqslant \Theta(t, \omega) \eta\left(|x-y|^{2}\right),
\end{aligned}
$$

then the stochastic differential equations (1) have the pathwise uniqueness.

Proof. Let $\eta_{t}=X_{t}-Y_{t}$ and $\xi_{t}=\eta_{t}^{2}$, where $(X, B)$ and $(Y, B)$ are the solutions of stochastic differential equations (1) with the same initial value. Let $\theta>0$; define the function $\Gamma_{\theta}:[0,1] \rightarrow$ $\mathbb{R}^{+}$as follows:

$$
\Gamma_{\theta}(x)=\int_{0}^{x} \frac{1}{\eta(s)+\theta} d s .
$$

It is obvious that, for any $0<s<1$, we have

$$
\Gamma_{\theta}(x) \uparrow \Gamma_{0}(x)=\int_{0}^{x} \frac{1}{\eta(s)} d s=\infty, \quad \text { as } \theta \downarrow 0 .
$$

Because of

$$
\begin{aligned}
& \frac{\Gamma_{\theta}\left(x_{2}\right)-\Gamma_{\theta}\left(x_{1}\right)}{x_{2}-x_{1}} \geqslant \frac{\Gamma_{\theta}\left(x_{3}\right)-\Gamma_{\theta}\left(x_{2}\right)}{x_{3}-x_{2}} \\
& 0<x_{1}<x_{2}<x_{3}<\epsilon,
\end{aligned}
$$

we get the conclusion that $\Gamma_{\theta}$ is a concave function on $[0, \epsilon)$. Letting

$$
\widetilde{\Gamma}_{\theta}(x)= \begin{cases}\Gamma_{\theta}(x), & x \in[0, \epsilon), \\ \text { a concave function, } & x \in \mathbb{R} \backslash[0, \epsilon),\end{cases}
$$

then $\Gamma_{\theta}^{\prime \prime}$ is a nonpositive random measure in the distributive sense.

Let $\tau=\inf \left\{t>0, \xi_{t} \geqslant \epsilon\right\}$; by Itô formula, we have

$$
\begin{aligned}
\widetilde{\Gamma}_{\theta}\left(\xi_{t \wedge \tau}\right)=\Gamma_{\theta}\left(\xi_{t \wedge \tau}\right)=1+2 \int_{0}^{t \wedge \tau} \Gamma_{\theta}^{\prime}\left(\xi_{s}\right) \\
\cdot\left\langle\eta_{s}, M\left(X_{s}, d s\right)-M\left(Y_{s}, d s\right)\right\rangle+2 \int_{0}^{t \wedge \tau} \Gamma_{\theta}^{\prime}\left(\xi_{s}\right) \\
\cdot\left\langle\eta_{s}, b\left(X_{s}, s\right)-b\left(Y_{s}, s\right)\right\rangle d s+\int_{0}^{t \wedge \tau} \Gamma_{\theta}^{\prime}\left(\xi_{s}\right) \\
\cdot\left[a\left(X_{s}, X_{s}, s\right)-2 a\left(X_{s}, Y_{s}, s\right)+a\left(Y_{s}, Y_{s}, s\right)\right] d s \\
+2 \int_{0}^{t \wedge \tau} \Gamma_{\theta}^{\prime \prime}\left(\xi_{s}\right) \eta_{s}^{2} \\
\cdot\left[a\left(X_{s}, X_{s}, s\right)-2 a\left(X_{s}, Y_{s}, s\right)+a\left(Y_{s}, Y_{s}, s\right)\right] d s .
\end{aligned}
$$

Notice that $\Gamma_{\theta}{ }^{\prime \prime}(\xi) \leqslant 0, \xi \in[0, \epsilon)$; applying hypothesis (25) and taking expectation for (30), we get

$$
\begin{aligned}
& \mathbb{E} \Gamma_{\theta}\left(\xi_{t \wedge \tau}\right) \leqslant 2 \mathbb{E} \int_{0}^{t \wedge \tau} \Gamma_{\theta}{ }^{\prime}\left(\xi_{s}\right) \\
& \cdot\left\langle\eta_{s}, b\left(X_{s}, s\right)-b\left(Y_{s}, s\right)\right\rangle d s+\mathbb{E} \int_{0}^{t \wedge \tau} \Gamma_{\theta}^{\prime}\left(\xi_{s}\right) \\
& \cdot\left[a\left(X_{s}, X_{s}, s\right)-2 a\left(X_{s}, Y_{s}, s\right)+a\left(Y_{s}, Y_{s}, s\right)\right] d s \\
& \leqslant \mathbb{E} \int_{0}^{t \wedge \tau} \frac{1}{\eta\left(\xi_{s}\right)+\theta} \Theta(s, \omega) \eta\left(\xi_{s}\right) d s \\
& \quad \leqslant \mathbb{E} \int_{0}^{t} \Theta(s, \omega) d s:=C_{t}<\infty .
\end{aligned}
$$

Letting $\theta \downarrow 0$ in inequality (31), we have $\mathbb{E} \int_{0}^{\xi_{t \wedge \tau}}(1 / \eta(s)) d s \leqslant$ $C_{t}<\infty$. It means that, for any $t$ given,

$$
\xi_{t \wedge \tau}=0 \text {, a.s. }
$$

If $P(\tau<+\infty)>0$, then there exists some $T>0$ big enough such that $P(\tau \leqslant T)>0$. By (32), for all $t \in \mathbb{Q} \cap[0, T], \xi_{t \wedge \tau}=$ 0 , a.s. It follows that, on $\{\tau \leqslant T\}, \xi_{\tau}=0$, which is absurd with the definition of $\tau$. Therefore $\tau=+\infty$ almost surely and, for any $t$ given, $\xi_{t}=0$ almost surely. By the continuity of samples, two solutions are indistinguishable.

Remark 3. The proved method of [15] is not suitable here. In fact, let $\Phi_{\theta}(\xi)=e^{\Gamma_{\theta}(\xi)}$; we get $\Phi_{\theta}^{\prime \prime}(\xi)=\Phi_{\theta}(\xi)((1-$ $\left.\left.\eta^{\prime}(\xi)\right) /(\eta(\xi)+\theta)^{2}\right)$; by (30) we know that

$$
\begin{aligned}
\int_{0}^{t \wedge \tau} \Phi_{\theta}^{\prime \prime}\left(\xi_{s}\right) d \xi_{s} \cdot d \xi_{s} \leqslant & \int_{0}^{t \wedge \tau} \Phi_{\theta}\left(\xi_{s}\right)\left|\frac{1-\eta^{\prime}\left(\xi_{s}\right)}{\left(\eta\left(\xi_{s}\right)+\theta\right)^{2}}\right| \\
& \cdot \eta\left(\xi_{s}\right) \cdot \xi_{s} d s .
\end{aligned}
$$

Notice that $\left|\left(1-\eta^{\prime}\left(\xi_{s}\right)\right) /\left(\eta\left(\xi_{s}\right)+\theta\right)^{2}\right| \cdot \eta\left(\xi_{s}\right) \cdot \xi_{s}$ may be unbounded, such that Gronwall lemma cannot be used here.

By the above pathwise uniqueness, we can also obtain the following continuity result.

Theorem 4. Suppose that the coefficients $a$ and $b$ satisfy assumptions (7) and (25). Let $X_{t}\left(x_{0}\right)$ be the solution of the SDE (1) with initial value $x_{0}$. Then, for any $\epsilon>0$, one has

$$
\lim _{y \rightarrow x} P\left(\sup _{0 \leqslant s \leqslant t}|X(s, y)-X(s, x)|>\epsilon\right)=0 .
$$

Proof. Consider $x$ and $y$ satisfying $|y-x|<\epsilon$; let

$$
\xi_{t}=\left|X_{t}(y)-X_{t}(x)\right|^{2} \text {. }
$$

Define $\tau(x, y)=\inf \left\{t>0, \xi_{t}>\epsilon^{2}\right\}$; let $\Gamma_{\theta}$ be the function defined in Theorem 2. By Itô formula, we have

$$
\mathbb{E} \Gamma_{\theta}\left(\xi_{t \wedge \tau(x, y)}\right) \leqslant \Gamma_{\theta}\left(\xi_{0}\right)+C_{t}, \quad \forall t>0, \theta>0,
$$


where $C_{t}$ is the same as in (31). Let $\theta=|x-y|$ in the above inequality; it holds that

$$
\mathbb{E} \Gamma_{\theta}\left(\xi_{t \wedge \tau(x, y)}\right) \leqslant \int_{0}^{\theta^{2}} \frac{1}{\eta(s)+\theta} d s+C_{t} \leqslant \theta+C_{t}
$$

so

$$
P(\tau(x, y)<t) \Gamma_{\theta}\left(\epsilon^{2}\right) \leqslant \mathbb{E} \Gamma_{\theta}\left(\xi_{t \wedge \tau(x, y)}\right) \leqslant \theta+C_{t} .
$$

Then we can obtain

$$
\begin{aligned}
& P\left(\sup _{0 \leqslant s \leqslant t}|X(s, y)-X(s, x)|>\epsilon\right) \\
& \quad=P(\tau(x, y)<t) \leqslant \Gamma_{\theta}^{-1}\left(\epsilon^{2}\right)\left(\theta+C_{t}\right) \longrightarrow 0
\end{aligned}
$$

as $\theta=|x-y| \rightarrow 0$. The proof is completed.

Remark 5. We should point out that the results (Theorems 1 and 2) of [15] are the special case of this paper result (Theorems 1 and 2). In fact, let $\gamma(x)=x \rho(x), \eta(x)=x \lambda(x)$ (where $\rho(x), \lambda(x)$ are the corresponding function of [15]); it is clear that $\gamma(x), \eta(x)$ satisfy conditions (7) and (25), respectively. Hence, the result of this paper includes the result of [15]. What is more, $\eta$ only need continuity in Theorem 2 ; however, $\lambda$ must be differential function in $[8,15]$, and our proof is more directive.

\section{Conflict of Interests}

The author declares that there is no conflict of interests regarding the publication of this paper.

\section{Acknowledgments}

The author deeply thanks the anonymous referees for their very kind and useful suggestions, which have improved the presentation of the paper. This work is supported by Ph.D. Research Start-Up Foundation (Grant no. 109-400211411) of Xian University of Technology. The author gratefully acknowledges the support.

\section{References}

[1] X. R. Mao, Exponential Stability of Stochastic Differential Equation, vol. 182 of Monographs and Textbooks in Pure and Applied Mathematics, Marcel Dekker, 1994.

[2] D. W. Stroock and S. R. Varadhan, Multidimensional Diffusion Processes, vol. 233 of Grundlehren der Mathematischen Wissenschaften, Springer, Berlin, Germany, 1979.

[3] N. V. Krylov, "On Itô's stochastic integral equations," Theory of Probability \& Its Applications, vol. 14, no. 2, pp. 330-336, 1969.

[4] N. Ikeda and S. Watanabe, Stochastic Differential Equations and Diffusion Processes, North Holland/Kodanska, 1989.

[5] D. Revuz and M. Yor, Continuous Martingales and Brownian Motion, vol. 293 of Grundlehren der mathematischen Wissenschaften, Springer, Berlin, Germany, 1991.

[6] P. Protter, Stochastic Integration and Differential Equations, Springer, Berlin, Germany, 1990.
[7] N. V. Krylov and M. Röckner, "Strong solutions of stochastic equations with singular time dependent drift," Probability Theory and Related Fields, vol. 131, no. 2, pp. 154-196, 2005.

[8] S. Fang and T. Zhang, "A study of a class of stochastic differential equations with non-Lipschitzian coefficients," Probability Theory and Related Fields, vol. 132, no. 3, pp. 356-390, 2005.

[9] X. Zhang, "Homeomorphic flows for multi-dimensional SDEs with non-Lipschitz coefficients," Stochastic Processes and Their Applications, vol. 115, no. 3, pp. 435-448, 2005.

[10] X. Zhang, "Strong solutions of SDES with singular drift and Sobolev diffusion coefficients," Stochastic Processes and their Applications, vol. 115, no. 11, pp. 1805-1818, 2005.

[11] A. M. Davie, "Uniqueness of solutions of stochastic differential equations," International Mathematics Research Notices. IMRN, vol. 2007, 2007.

[12] W. Y. Fei, "Existence and uniqueness of solution for fuzzy random differential equations with non-Lipschitz coefficients," Information Sciences, vol. 177, no. 20, pp. 4329-4337, 2007.

[13] D. Luo, "Regularity of solutions to differential equations with non-Lipschitz coefficients," Bulletin des Sciences Mathématiques, vol. 132, no. 4, pp. 257-271, 2008.

[14] G. Q. Lan, "Pathwise uniqueness and non-explosion of SDEs with non-Lipschitzian coefficients," Acta Mathematica Sinica, Chinese Series, vol. 52, no. 4, 2009.

[15] W. Y. Fei, "Uniqueness of solutions to SDEs driven by semimartingle with non-lipschitz conditions," Journal of Mathematics, vol. 30, 2010.

[16] G. L. Cao, "Existence and pathwise uniqueness of solutions of SDE driven by a classs of special semimartingle," Journal of Graduate of Chinese Academay of Sciences, vol. 28, no. 1, 2011.

[17] X. Yang, "Pathwise uniqueness for an SPDE with jumps and non-Lipschitz coefficienties," Journal of Beijing Normal University (Natural Science), vol. 50, no. 3, 2014.

[18] A. Friedman, Stochastic Differential Equations and Applications, vol. 1, Academic Press, New York, NY, USA, 1975. 


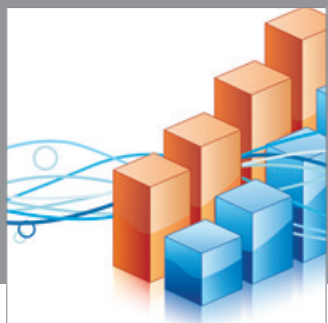

Advances in

Operations Research

mansans

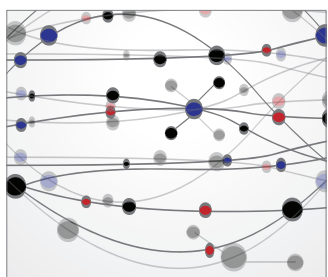

The Scientific World Journal
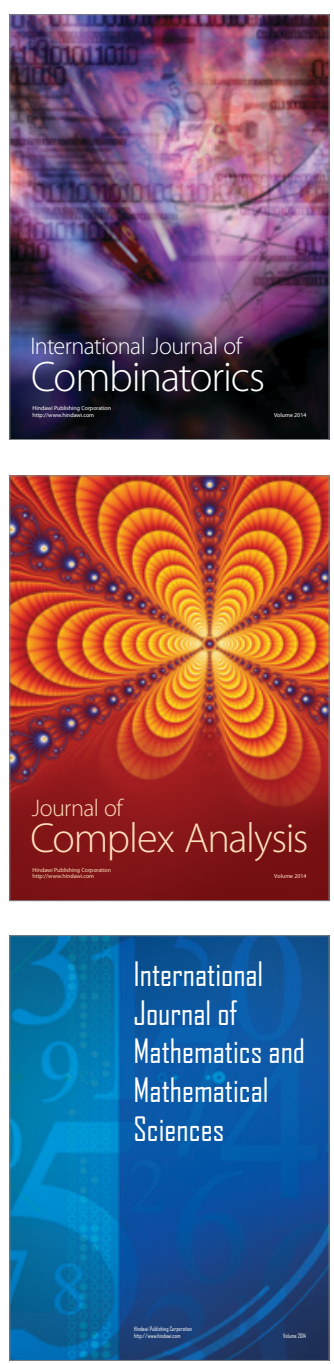
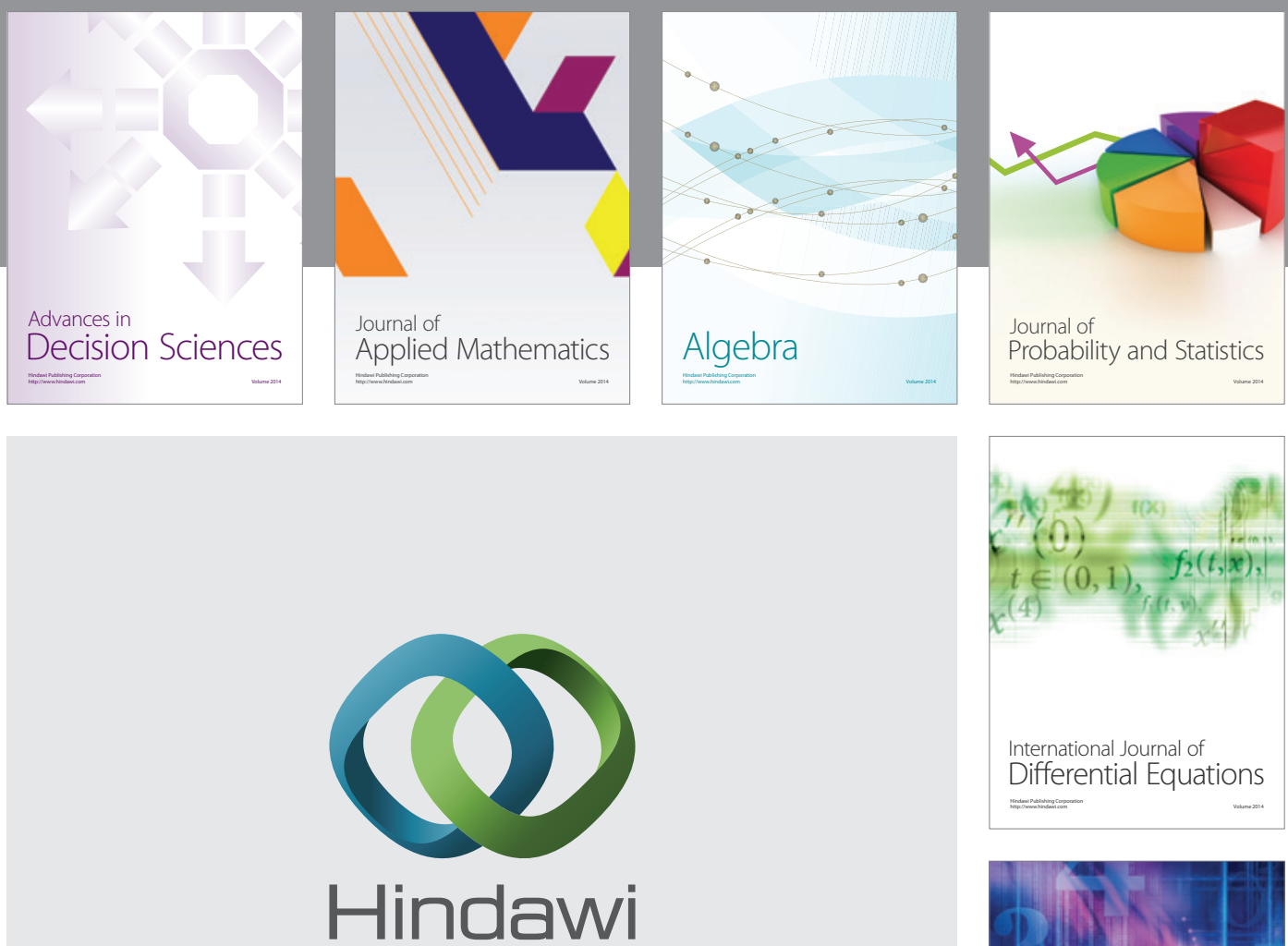

Submit your manuscripts at http://www.hindawi.com
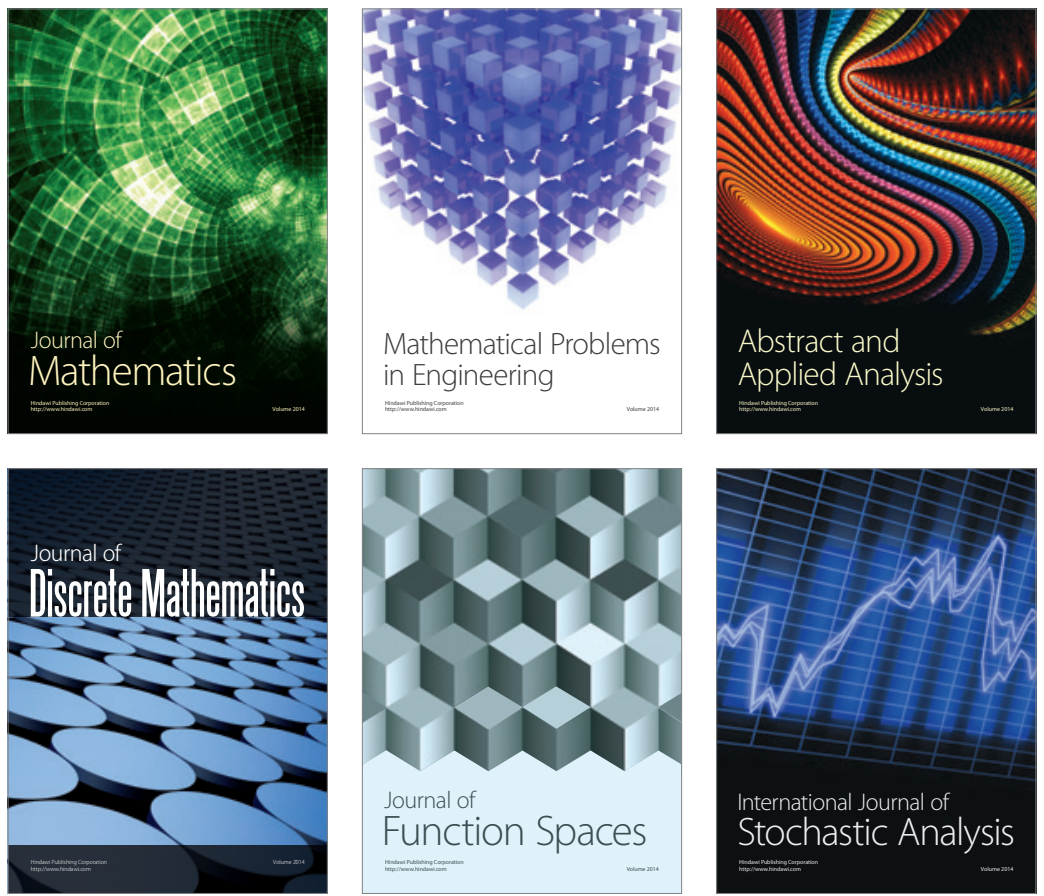

Journal of

Function Spaces

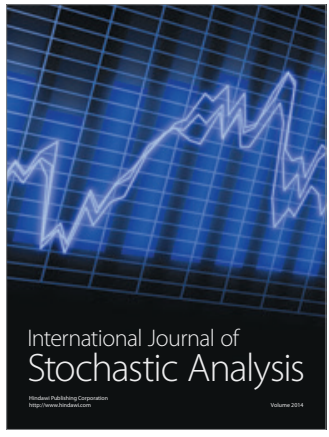

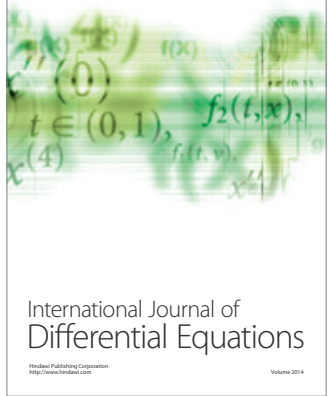
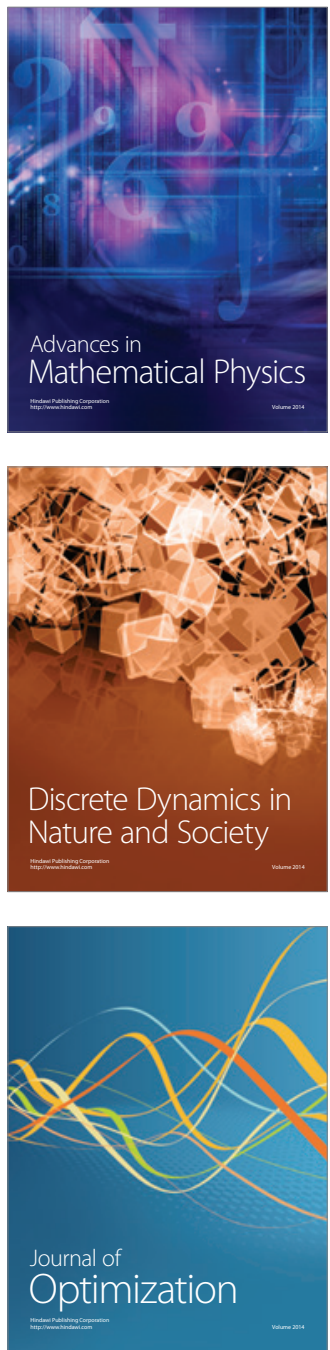